\title{
A useful method to keep azygos arch in minimally invasive resection of esophageal leiomyoma
}

\author{
Shaobin Yu ${ }^{1}$, Shuchen Chen ${ }^{1}$, Ziyang Han ${ }^{1}$, Sui Chen ${ }^{1}$, Mingduan Chen ${ }^{1}$, Jinghong Lin ${ }^{1}$, Shijie Huang ${ }^{1}$, \\ Lei Gao ${ }^{1}$, Mingqiang Kang ${ }^{1,2,3}$ \\ ${ }^{1}$ Department of Thoracic Surgery, Fujian Medical University Union Hospital, Fuzhou 350001, China; ${ }^{2}$ Key Laboratory of Ministry of Education for \\ Gastrointestinal Cancer, ${ }^{3}$ Fujian Key Laboratory of Tumor Microbiology, Fujian Medical University, Fuzhou 350122, China \\ Correspondence to: Mingqiang Kang, MD, PhD. Department of Thoracic Surgery, Fujian Medical University Union Hospital, Fuzhou 350001, China. \\ Email: kmq2015@163.com.
}

\begin{abstract}
Lifting azygos arch with a prolene line is a useful way to keep azygos arch in minimally invasive resection of esophageal leiomyoma without affecting surgical exposure and operative procedure.
\end{abstract}

Keywords: Esophageal leiomyoma; minimally invasive resection; vertically suspending azygos arch

Submitted Apr 27, 2017. Accepted for publication Aug 24, 2017.

doi: $10.21037 /$ jtd.2017.09.152

View this article at: http://dx.doi.org/10.21037/jtd.2017.09.152

\section{Introduction}

Esophageal leiomyoma arising from smooth muscle is the commonest benign esophageal tumor, which is most often found in the middle and lower thirds of the esophagus (1). Leiomyoma is more commonly seen in male with a male to female ratio of $2: 1$. It usually affects patients at age between 20 and 50 years (2). According to the tumor size and tumor location, different treatments are performed. Many scholars consider cutting azygos vein will benefit surgical exposure and facilitate operation for the middle-thirds leiomyoma resection. We recommend a useful kind of surgical skill, which can keep the azygos vein without affecting surgical exposure and operative procedure.

\section{Operative techniques}

A 61-year-old male with no history of dysphagia and chest pain had a medical examination. Gastroscopy showed a $0.8 \times 0.8 \mathrm{~cm}$ mass arising along back wall of esophagus and locating in $25 \mathrm{~cm}$ from the incisors. After eighteen months, endoscopic ultrasound (EUS) of esophagus revealed a $2.1 \times 1.0 \mathrm{~cm}$ submucosal mass arising from the fourth layer of esophageal wall and suggested leiomyoma of esophagus. A computed tomography (CT) scan of the chest revealed a mass in the middle thirds of the esophagus, which measured
$1.8 \mathrm{~cm}$ in maximum diameter and had a well-defined border (Figure 1). A meglumine diatrizoate esophagogram demonstrated esophageal had oval filling defect in the sixth thoracic vertebra level, which edged neatly around the normal mucosa. Laboratory examinations were normal. Surgical resection was suggested because the tumor size of the patients was obviously increased at the follow-up period and the diagnose was uncertain. The patient gave consent to the surgical treatment. Written informed consent was obtained from the patient for publication of this case report and any accompanying images.

We preferred to ventilate through the left lung and expose the tumor through the right side of the chest. The camera port $(1.5 \mathrm{~cm})$ was placed at the seventh intercostal space, middle axillary line. A $2-\mathrm{cm}$ port was placed in the anterior axillary line at the fourth intercostal space, which was used to expose surgical field. A 2-cm port for the ultrasonic shears was placed at the eighth intercostal space, posterior axillary line. The last $0.5-\mathrm{cm}$ port was placed in the posterior axillary line at the sixth intercostal space and used for retraction and countertraction by the surgeon. Right chest without effusion, smooth pleural without nodules, right lung adhesions visceral pleural adhesions. The azygos vein overlay the tumor. The surgeon used an ultrasound knife to cut the surface of the tumor mediastinal pleura, and then proceed to the upper edge of the odd vein 


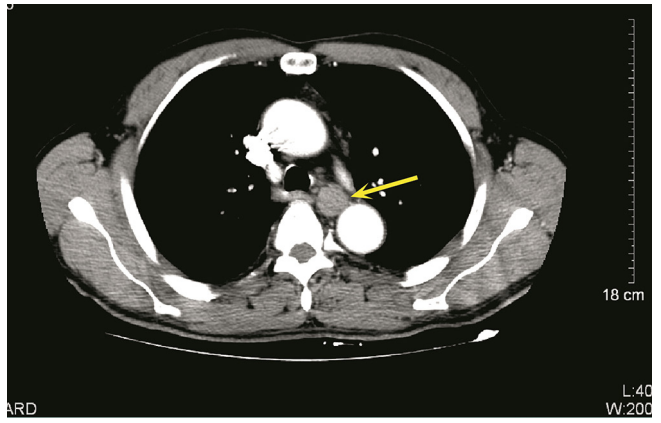

Figure 1 The CT scan for the patient of esophageal leiomyoma (the yellow arrow indicates the location of tumor).

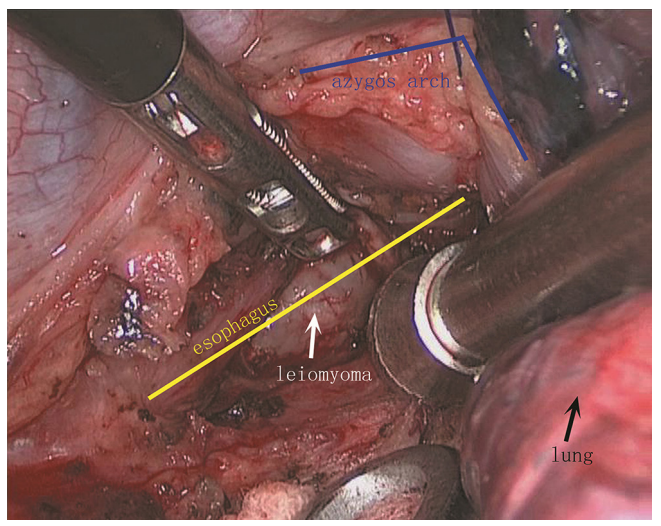

Figure 2 Vertically suspending azygos arch is a useful way to keep azygos arch in minimally invasive resection of esophageal leiomyoma.

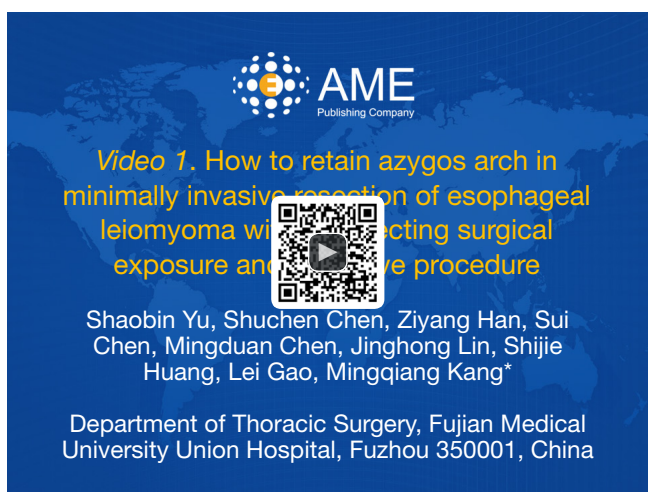

Figure 3 This video shows how to retain azygos arch in minimally invasive resection of esophageal leiomyoma without affecting surgical exposure and operative procedure (3).

Available online: http://www.asvide.com/articles/1837 arch. After the azygos vein was fully freed, 4-0 prolene line was threaded through the azygos vein. ENDO CLOSE needle (product number: T173022, COVIDIEN company) was inserted in the posterior axillary line at the fourth intercostal space to catch the prolene line and bring the prolene line out of the chest. After that, the end of prolene line was pulled to vertically sling the azygos vein and fixed on a sterile surgical towel (Figure 2). Thus, the surgical field below the azygos vein could be fully exposed. The surgeon used the ultrasonic knife to open the esophageal fibrous membrane, muscular. The tumor was separated from esophageal mucosa with blunt dissection using "pea nut" which was made with sterile gauze. After checking bleeding and the integrity of esophageal mucosa, the esophageal muscular layer and the mediastinal pleura were closed with interrupted sutures to prevent postoperative diverticular formation. We provided a surgery video containing detailed procedure for you to refer to (Figure 3).

After surgery, digital gastrointestinal imaging showed no esophageal thoracic cavity fistula. The final pathologic examination demonstrated that the mass was leiomyoma. The results of immunohistochemical stains showed positive stains for smooth muscle actin and Desmin and negative stains for CD117, CD34 and DOG-1. Besides, Ki-67 was $1 \%$ positive stains. The stomach tube was extubated on the third day, and the patient started a liquid diet. The patients could have normal diets with no symptom of dysphagia and regurgitation three months later.

\section{Comments}

It was reported that about $30 \%$ to $33 \%$ of esophageal leiomyomas were found in the middle thoracic esophagus $(4,5)$. Digital gastrointestinal imaging, thoracic CT and EUS are need to be performed to identify the tumor type. Minimally invasive techniques have become leading approaches to remove esophageal leiomyoma for decreasing postoperative complications, improving postoperative pain control and shorting hospital stays (4). For leiomyoma in the middle thirds of the esophagus, cutting azygos vein in minimally invasive surgeries has been recommended in many articles, because it can benefit surgical exposure and facilitate operations in resecting leiomyoma $(1,5)$. However, cutting azygos vein alters venous circulation. And the instrument is needed during operation to push the azygos vein arch stub away for a better surgical exposure. We indicate a useful surgical technique, vertical sling of the azygos vein, which can fully expose surgical field without 
cutting azygos vein and make adding the instrument to open the azygos vein arch stub become unnecessary. Excessive pulling of the azygos vein may increase the risk of venous tear. Besides, azygos vein arch can be covered on the surface of esophageal longitudinal incision after resection of esophageal leiomyoma which may reduce the exposure and promote the healing of esophageal longitudinal incision.

\section{Acknowledgements}

Funding: This study was supported by Fujian Medical Innovation Fund (2014-CX-15), Fujian Young Teacher Fund (JAT160209), Sailing Fund of Fujian Medical University (2016QH036).

\section{Footnote}

Conflicts of Interest: The authors have no conflicts of interest to declare.

Informed Consent: Written informed consent was obtained from the patient for publication of this case report and any accompanying images.

\section{References}

1. Ben-David K, Alvarez J, Rossidis G, et al. Thoracoscopic and Laparoscopic Enucleation of Esophageal Leiomyomas. J Gastrointest Surg 2015;19:1350-4.

2. Diego R, Pablo P, Magdalena C, et al. Comparative study between open and minimally invasiveapproach in the surgical management of esophageal leiomyoma. Rev Esp Enferm Dig 2016;108:8-14.

3. Yu S, Chen S, Kang M, et al. How to retain azygos arch in minimally invasive resection of esophageal leiomyoma without affecting surgical exposure and operative procedure. Asvide 2017;4:518. Available online: http:// www.asvide.com/articles/1837

4. Ha C, Regan J, Cetindag IB, et al. Benign esophageal tumors. Surg Clin North Am 2015;95:491-514.

5. Mujawar P, Pawar T, Chavan RN. Video Assisted Thoracoscopic Surgical Enucleation of a Giant Esophageal Leiomyoma Presenting with Persistent Cough. Case Rep Surg 2016;2016:7453259.
Cite this article as: Yu S, Chen S, Han Z, Chen S, Chen M, Lin J, Huang S, Gao L, Kang M. A useful method to keep azygos arch in minimally invasive resection of esophageal leiomyoma. J Thorac Dis 2017;9(11):4589-4591. doi: 10.21037/ jtd.2017.09.152 